Methods From 2001 to 2016, incidence rates of cervical cancer were estimated from United States Cancer Statistics. SEER*Stat and Joinpoint regression were used to calculate the incidence rate (per 100,000) and average annual percent change (AAPC), adjusted for hysterectomy and pregnancy prevalence data from the Behavioral Risk Factor Surveillance System.

Results Adenosquamous cell carcinoma accounted for 6,599 of $200,000(3.3 \%)$ cases of cervical cancer from 2001-2016; of which 4,165 were White (63.1\%), 830 were Black (12.6\%), 1,155 were Hispanic (17.5\%), 345 were Asian (5.2\%) and 104 were unidentified (1.6\%). There was a $3.9 \%$ decrease in incidence per year; from $0.47 / 100,000$ in 2001 to 0.24 / 100,000 in 2016 after adjusting for age and race $(p<0.001)$. The incidence of ASC is nearly 1.5 -fold greater for Hispanics at 0.38 per 100,000 compared to Whites $(0.25)$ and Blacks (0.27). Additionally, the Hispanics had a bimodal age distribution at diagnosis, with peaks at 40-44yo and 65-69yo $(0.76$ and 0.69$)$ compared to Whites with a single peak diagnosis at 40-44yo $(0.56 / 100,000)$.

\section{INCIDENCE OF ADENOSQUAOUS} CARCINOMA BY RACE

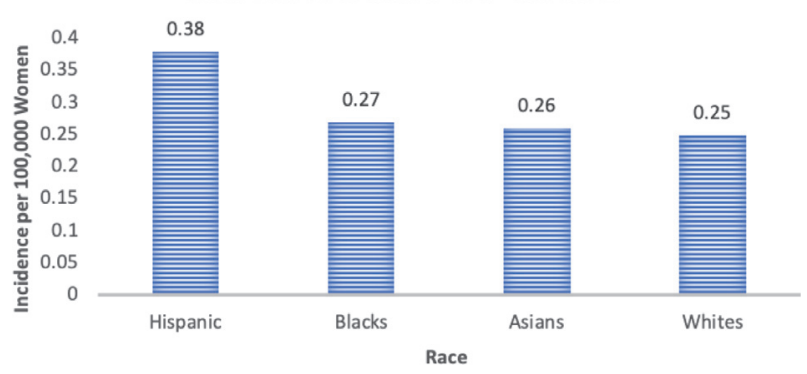

Abstract 141 Figure 1

Conclusion Hispanics have a 50\% higher incidence of adenosquamous cell cervical carcinoma compared to others. Their incidence peaks in the early 40's and late 60's year olds. The difference in incidence and age distribution of this cancer warrant further investigation.

\section{IGCS20 1119}

\section{SURGEON-ADMINISTERED ILIO-INGUINAL AND PUDENDAL NERVE BLOCKS FOR VULVAL ONCOLOGY SURGERY: AN EVALUATION WITH VISUAL ANALOGUE PAIN SCORING}

P Maguire*, Y Shahabuddin, N Gleeson. Department of Gynaecological Oncology, St James's Hospital, Ireland

\subsection{6/ijgc-2020-IGCS.122}

Introduction Surgery for vulval cancer includes sampling diagnostic, excisional biopsy and extensive radical surgery. The vulva and perineum are innervated by branches from the ilioinguinal and pudendal nerves. We describe our experience of

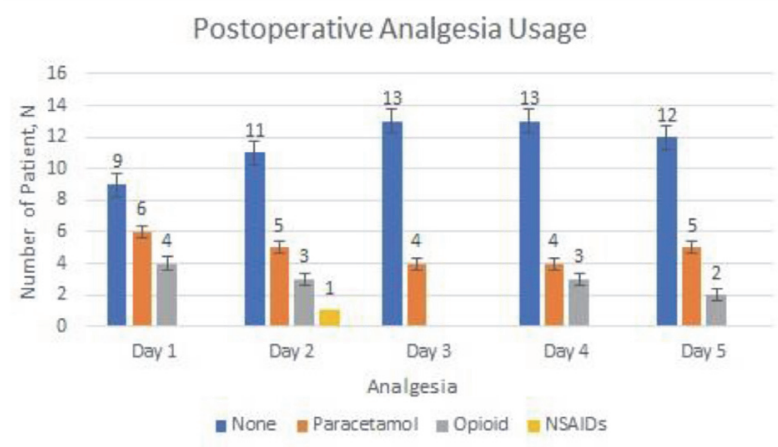

Abstract 142 Figure 1

axon nerve blocks and outcomes including postoperative pain scores following surgeon administered intraoperative ilio-inguinal and pudendal nerve blocks.

Methods Ilio-inguinal and pudendal nerve block has become routine practice for women undergoing vulval surgery in our cancer centre. In a retrospective chart review, clinical and demographic data, postoperative visual analogue pain scores and use of analgesia were recorded.

Results Eighteen women were included in the analysis. Their median age was 67 (range 34-81) years and thirteen (72\%) were over 60 years. Visual analogue scores ranged from 0 to 3 for 17 patients from day $0-1$ and 15 patients from days 25. Two patients had pain scores $>4$ on one or more postoperative day: one had chronic arthralgia and one had received a lower volume of bupivicaine compared to our routine practice. Figure 1 summarizes postoperative analgesia usage for the 18 women.

Conclusion Ilio-inguinal and pudendal nerve block is a feasible and effective strategy for postoperative pain management in women undergoing vulval surgery.

\section{IGCS20 1120}

\section{DEVELOPMENT AND CLINICAL APPLICATION OF A TOOL TO IDENTIFY FRAILTY IN ELDERLY PATIENTS WITH GYNECOLOGICAL CANCERS}

A Ferrero*, M Villa, D Attianese, M Coppo, M Borghese, E Badellino, L Fuso, N Biglia. Academic Department Gynaecology and Obstetrics - Mauriziano Hospital, Italy

\subsection{6/ijgc-2020-IGCS.123}

Objectives Aim of this prospective study is the development and clinical application of a tool to identify frailty in patients $>70$ years old affected by either ovarian or endometrial cancer. After identifying a cut off to establish frailty, differences in terms of surgical complications and chemotherapy toxicities were verified.

Methods The test consists of 20 items combining comorbidities and functional aspects. At the onset or at the first recurrence 52 patients were evaluated before treatment's administration.

Results Considering 'completion of treatment' as parameter to discriminate frail patients, a cut off $>4$ resulted the best in 